\title{
Editorial: Muscle Mechanics, Extracellular Matrix, Afferentation, Structural, and Neurological Coupling and Coordination in Health and Disease
}

\author{
Can A. Yucesoy ${ }^{1 *}$, Eva Pontén ${ }^{2}$, Francisco J. Valero-Cuevas ${ }^{3}$, Mark Smeulders ${ }^{4}$ and \\ Ciaran Knut Simms ${ }^{5}$ \\ ${ }^{1}$ Institute of Biomedical Engineering, Bogaziçi University, Istanbul, Turkey, ${ }^{2}$ Karolinska Institutet, Stockholm, Sweden, \\ ${ }^{3}$ University of Southern California, Los Angeles, CA, United States, ${ }^{4}$ Zuyderland Medical Centre, Sittard, Netherlands, ${ }^{5}$ Trinity \\ College Dublin, Dublin, Ireland
}

Keywords: muscle mechanics, extracellular matrix, connective tissue, neuromusculoskeletal interaction, adaptation, pathology, force production - transmission phenomena

\section{Editorial on the Research Topic}

Muscle Mechanics, Extracellular Matrix, Afferentation, Structural, and Neurological Coupling and Coordination in Health and Disease

\section{OPEN ACCESS}

Edited and reviewed by:

Paul M. L. Janssen,

The Ohio State University,

United States

*Correspondence:

Can A. Yucesoy

can.yucesoy@boun.edu.tr

Specialty section:

This article was submitted to

Striated Muscle Physiology,

a section of the journal

Frontiers in Physiology

Received: 26 October 2021 Accepted: 03 November 2021 Published: 06 December 2021

Citation:

Yucesoy CA, Pontén E

Valero-Cuevas FJ, Smeulders $M$ and Simms CK (2021) Editorial: Muscle Mechanics, Extracellular Matrix,

Afferentation, Structural, and Neurological Coupling and Coordination in Health and Disease.

Front. Physiol. 12:802202.

doi: 10.3389/fphys.2021.802202
There is a growing emphasis on the importance of muscle extracellular matrix on muscular mechanics and specifically on the effects of the interaction of the extracellular matrix (ECM) and the contractile apparatus. Accordingly, investigation of the role of muscle extracellular matrix and other myofascial tissues continuous with the epimysium on muscle force production was one of the main aims of this Research Topic. From the muscle function viewpoint, the structure and role of intramuscular connective tissue was discussed in a comprehensive review (Purslow). This work placed an emphasis on the shearing of intramuscular connective tissues as the mechanism coordinating forces and displacements between adjacent muscle cells and indicated the role of this in myofascial force transmission (e.g., Huijing, 2009; Yucesoy, 2010). With the aforementioned mechanical interaction accounted for as a key determinant, experimental, and computational quantification assessments for muscle fiber direction length changes or direct sarcomere length changes were also an aim for this Research Topic. An experimental assessment using multi-photon excitation microscopy in mouse tibialis anterior muscle surgically dissected from the tibia and surrounding muscles showed that in isometrically activated muscle the sarcomeres re-organize their lengths into a more uniform pattern over time, whereas in the passive state their length non-uniformity remained the same (Moo and Herzog). Sarcomere lengths in isolated mice soleus muscle in vivo were quantified using a force microscope by minimally imaging 20 continuous sarcomere segments, which were used for length measurements (Tsai et al.). Samples from animals exposed to different durations of ischemic stroke suggested that sarcomere additions were absent, with implications for muscle spasticity and/or joint contracture that may follow stroke. Magnetic resonance imaging (MRI) is a powerful tool (Figure 1) to quantify local muscle tissue displacements and strains (e.g., Blemker et al., 2007; Pamuk and Yucesoy, 2015) and tissue structure (e.g., Sinha et al., 2006), while along with continuum-based computational modeling (e.g., Böl and Reese, 2008; Yucesoy and Huijing, 2012) and novel modeling concepts and approaches (see below), these techniques can allow the development of new phenomena (e.g., Cankaya et al., 2021), 


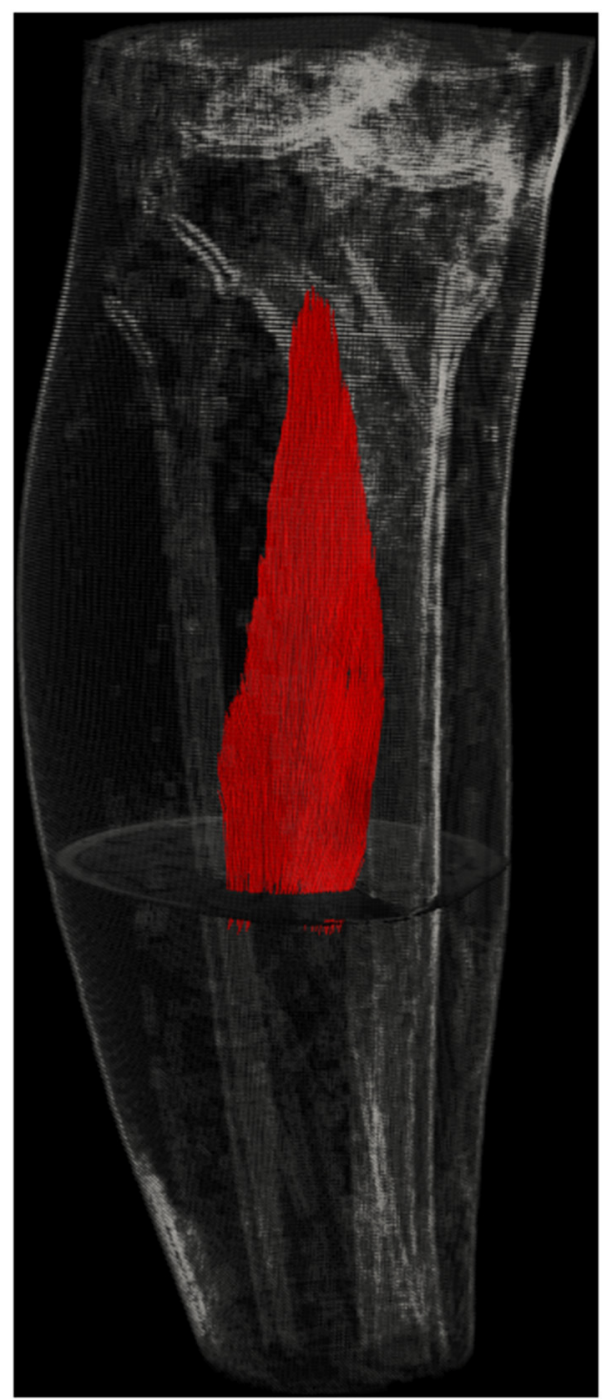

FIGURE 1 | Visualization of human muscle using MRI and DTI. Utilization of advanced imaging techniques offers major potential for analysis of structure as well as local muscle length and shape changes for human muscles in vivo.

addressing clinical problems (Westman et al., 2019; Marty and Carlier, 2020; Ramasamy et al.) and understanding mechanisms of treatments (Yucesoy and Huijing, 2009; Turkoglu and Yucesoy, 2016). A review (Sinha et al.) focused on several aspects of advanced MRI analyses, diffusion tensor imaging (DTI), and modeling to quantify muscle tissue strain rates, strains, and fiber orientations to study the effects of aging and disuse-related remodeling of the extracellular matrix on force transmission in the human musculoskeletal system. The authors indicated, in collaboration with Purslow, shearing in the endomysium as a marker for myofascial force transmission, and muscle fiber shear strains for that purpose were quantified recently in human muscle in vivo (Pamuk et al., 2016). Although, earlier Street (1983) and recently others (e.g., Purslow; Malis et al.) referred to the mechanical role played by the multimolecular transsarcolemmal connections between the endomysium and muscle fibers as lateral force transmission, the term lateral, may not be quite accurate as it suggests force is transmitted perpendicularly to the muscle fiber surface. Several studies report sarcomere length heterogeneity (Pappas et al., 2002; Lichtwark et al., 2018; Moo et al.), which according to myofascial force transmission phenomena is ascribed to myofascial loads (Yucesoy, 2010), i.e., forces acting locally along the muscle fiber originating (i) intramuscularly from the ECM and sarcomeres in neighboring muscle fibers and (ii) epimuscularly from muscle connections to surrounding muscles and other tissues. These forces can affect the mechanical equilibrium determining sarcomere lengths and are aligned with the sarcomeres rather than being normal to them. The principles of this mechanism were studied as a part of this Research Topic using finite element modeling and by comparing isolated muscle with muscle modeled within the framework of its surrounding tissues (Pamuk et al.). The results exemplified heterogeneity of muscle fiber direction length changes occurring in context with myofascial loads acting along the muscle fibers. Two of the three cases studied were designed to represent principles of previous MRI and DTIbased experiments conducted in humans, in vivo (Pamuk et al., 2016; Karakuzu et al., 2017). They demonstrate how a passively lengthened muscle can also have shortened parts and how an isometric contracting muscle can also have lengthened parts along the muscle fascicles. In another study in vivo, published as a part of this Research Topic, the effects of passive ankle dorsiflexion imposed by an isokinetic dynamometer on human semimembranosus muscle were assessed using high-resolution ultrasound (Wilke and Tenberg, 2020). The authors reported muscle tissue displacement in the dorsal thigh, despite the fact that knee angle was kept constant, and explained this with myofascial force transmission across the knee joint. Such mechanical interaction via the fascial system (Adstrum et al., 2017; Stecco et al., 2018; Wilke et al., 2018) between muscles within the same limb segment (e.g., Huijing et al., 2011; Marinho et al., 2017; Ateş et al., 2018a) and even across segments, e.g., in the gastrocnemius after an imposed anterior pelvic tilt (CruzMontecinos et al., 2015) have been reported in numerous studies. In fresh postmortem human subjects, strain mechanisms in lower limb deep fascia induced by passive knee movement were studied using motion analysis and digital image correlation techniques (Sednieva et al.). Shedding light on fascia/muscle interactions, for anterior and lateral fascia, tension, and for the iliotibial tract, dependent knee movement, tension, or pure shear mechanisms were observed in extended or flexed positions, respectively. Based on previous evidence reported on coupling between fascia and skeletal muscle shown in fundamental/animal studies (Huijing, 1999; Huijing and Jaspers, 2005; Stecco et al., 2013; Wilke et al., 2018), using ultrasound (Bojsen-Møller et al., 2010; Tian et al., 2012; Le Sant et al., 2017; Ateş et al., 2018a), using MRI (Huijing et al., 2011; Pamuk et al., 2016; Karakuzu et al., 2017), and intraoperative work (Smeulders et al., 2004, 2005; Smeulders and Kreulen, 2007; de Bruin et al., 2014; Kaya et al., 2019, 2020). Zullo et al. reviewed the functional effects of aging on the neuromusculoskeletal system. They discussed occurrence of loss of effectiveness in the locomotory apparatus, due to the molecular and cellular changes occurring in the myofascia, the skeletal 
muscle tissue, the nervous system, and their structural and functional coupling.

\section{NOVEL COMPUTATIONAL APPROACHES FOR DIFFERENT SCALES OF NEURO-MUSCULO-SKELETAL MECHANICS}

Understanding the individual contributions of ECM and skeletal muscle fibers to overall passive muscle stiffness presents a particular challenge; Wheatley has used a combination of experimental and computational approaches to show that biaxial tissue testing can provide additional insight over uniaxial test results, and further demonstrate how ECM collagen helps to determine passive muscle stiffness. Klotz et al. discuss fundamental challenges with evaluating detailed constitutive models of skeletal muscle as internal variables are difficult to experimentally quantify, and model predictions at the local muscle level are difficult to verify. As such, these detailed computational models might be considered as philosophical explorations, and their main utility may be to guide the planning of future experimental works. Wakeling et al. and Ryan et al. address energy storage in skeletal muscle, how shape changes in the muscle result in energy storage even without overall length changes, and how some contractile energy is lost in overcoming passive resistance to longitudinal contraction and accompanying lateral expansion due to the largely incompressible nature of the tissue. Ross et al. extend the work of Wakeling et al. and Ryan et al. to dynamic contractions and show how relative energy storage in the aponeurosis increases compared to energy storage in the ECM. Taken together, these papers substantially strengthen our understanding of muscle mechanics.

\section{CLINICAL IMPLICATIONS: MECHANISMS OF PATHOLOGICAL CONDITIONS AND TREATMENT TECHNIQUES WITH A SPECIAL EMPHASIS ON SPASTIC CEREBRAL PALSY}

Cerebral palsy (CP) is defined as decreased motor control due to an injury to the developing brain, before evolvement of mature gait or fine motor skills. Motor control is simplified, and children use simpler muscle synergies while walking compared to typically developed (TD) children. However, treatment for gait improvement only marginally changes muscle synergies (Bekius et al.). Typically, the children are born hypotonic, with a normal range of motion of the joints, but will eventually develop contractures of the joints due to both spasticity and shortening of the musculotendinous complex (Hedberg-Graff et al., 2019; Lindén et al., 2019). At the age of about 9, 3D ultrasound of the medial gastrocnemius muscle and the Achilles tendon has showed that in $\mathrm{CP}$ the tendon is relatively longer compared to in TD children, and related to a larger stiffness of the muscle in CP (Weide et al.). Muscle shortening in conjunction with spasticity will force the ankle into flexion, and the heel does not reach the floor when walking, i.e., the children walk with equinus. Proper understanding of the mechanical effect of overactive plantar flexors is critical to clinical diagnosis and treatment, but it can be underestimated if the Achilles tendon moment arm is overestimated by assuming a straight line of action (instead of its actual curved path) (Harkness-Armstrong et al.). One way of diminishing the spasticity is selective dorsal rhizotomy, which by instrumented gait analysis has shown short-term diminishing effects on so-called quasi-stiffness (i.e., the slope of the ankle moment vs. the ankle angle plot) of the calf muscles (Ates et al.). Another local and temporary way of diminishing toe walk due to spasticity is by botulinum toxin (BTX) injection into the calf muscles. Assessments of serial biopsies from the gastrocnemius in CP in humans comparing BTX with saline has as yet not been published, but injections into the tibialis anterior in the nonspastic rat has shown positive acute effects (Ateş and Yucesoy, 2014; Yucesoy et al., 2015). After 1 month, atrophy and decreased active force of all muscles in the anterior compartment of the lower leg was evident (Kaya et al.). There was an increased content of hydroxy-proline in the BTX group compared to rats injected with only saline. One key biomechanical finding of animal studies noteworthy of clinical testing is that the BTX group also showed elevated muscle passive forces (e.g., Kaya et al.). Another potentially functionally important biomechanical finding shown in animal studies was a decrease in length range of force production reported for muscles exposed to BTX (Kaya et al.; Yilmaz et al., 2021). Therefore, studies on what happens before and after botulinum toxin injections in the muscle in children with $\mathrm{CP}$ is highly warranted. A new method developed based on percutaneous muscle microbiopsies is promising (Corvelyn et al.), and can, with repeated biopsies during growth, hopefully in the future be used in understanding the natural history of muscles in CP. The gait may be improved if the calf muscle is lengthened by Achilles tendon lengthening, which will increase the relative length of the already long tendon even further. Here, there is a great risk of future weakness of the calf muscles if both legs are affected, as in spastic diplegia, and the child might develop crouch gait. A more conservative and safer operative method is to just release the gastrocnemius from the soleus fascia and sometimes also release the soleus aponeurosis. Few experimental studies have been conducted on what actually happens when an aponeurosis is cut (Brunner et al., 2000; Jaspers et al., 2002; Ateş et al., 2013). The mechanism of acute effects assessed computationally (Yucesoy and Huijing, 2009) showed that major sarcomere shortening in muscle part distal to a cut leads to a decreased muscle force, and overall increased sarcomere length heterogeneity causes muscle peak force production to move to a longer muscle length. Brunner et al. investigated aponeurotic release of the gastrocnemius in the rat. They found that the length of the muscle complex was increased, and the cut and resulting defect of the aponeurosis was filled with more compliant scar tissue (Rivares et al.). This is highly important since it suggests that the above mentioned acute aponeurotic release effects may also prevail after recovery, which needs to be tested in patients.

Regarding the force transmitting role of the epimysial connective tissue in patients with $\mathrm{CP}$, active muscle length-force 
characteristics measured intraoperatively directly at the muscle tendon were shown to be affected by applying varying stretching on adjacent tissues of the tested spastic muscle (Smeulders et al., 2005). Likewise, mere distal tenotomy of the spastic flexor capri ulnaris muscle did not eliminate its contribution to the wrist flexion moment when epimuscular connective tissues were left intact (de Bruin et al., 2011), implying that the perimuscular connective tissues other than the proximal and distal tendon are important in transmitting force from a muscle to their target joints and structures. The role of collagen-reinforced connective tissues in the limited joint motion in spastic CP is still open to debate (de Bruin et al., 2013, 2014). Due to a highly variable distribution of intramuscular collagen content within muscle, the amount collagen content itself does not relate to the actual passive muscle properties during mechanical testing as was shown in mouse hind limb muscles (Binder-Markey et al.). Experimental work on rabbit lower leg muscles showed that passive load bearing properties of different muscles do vary. More importantly, mechanical testing of isolated muscle fibers compared, respectively, to bundles, fascicles, and whole muscle implied that the relative contribution to passive resistance changes from intracellular titin in isolated fibers to the extracellular perimysium in whole muscles (Binder-Markey et al.). However, in vivo, passive stiffness of monoarticular lower leg muscles was shown to be influenced by knee angle indicating intermuscular interactions may change muscular mechanics beyond a single muscle level. Note that, in $\mathrm{CP}$ patients, intraoperative testing of spastic semitendinosus and gracilis muscles did not show high passive forces measured at their tendon as a function of knee joint angle (Kaya et al., 2019, 2020). This suggests that in CP rather than the passive state, the active state is the determinant of the pathological condition, and co-activation of synergistic (Kaya et al., 2018) and also antagonistic muscles (Ateş et al., 2018b) was shown to elevate the active knee flexor force of individual spastic hamstrings significantly. Therefore, in the context of issues addressed in this Research Topic, mainly "the role of the muscle extracellular matrix and other myofascial tissues continuous with

\section{REFERENCES}

Adstrum, S., Hedley, G., Schleip, R., Stecco, C., and Yucesoy, C. A. (2017). Defining the fascial system. J. Bodyw. Mov. Ther. 21, 173-177. doi: 10.1016/j.jbmt.2016.11.003

Ateş, F., Andrade, R. J., Freitas, S. R., Hug, F., Lacourpaille, L., Gross, R., et al. (2018a). Passive stiffness of monoarticular lower leg muscles is influenced by knee joint angle. Eur. J. Appl. Physiol. 118, 585-593. doi: 10.1007/s00421-018-3798-y

Ateş, F., Özdeşlik, R. N., Huijing, P. A., and Yucesoy, C. A. (2013). Muscle lengthening surgery causes differential acute mechanical effects in both targeted and non-targeted synergistic muscles. J. Electromyography Kinesiol. 23, 1199-1205. doi: 10.1016/j.jelekin.2013. 05.010

Ateş, F., Temelli, Y., and Yucesoy, C. A. (2018b). Effects of antagonistic and synergistic muscles' co-activation on mechanics of activated spastic semitendinosus in children with cerebral palsy. Hum. Mov. Sci. 57, 103-110. doi: 10.1016/j.humov.2017.11.011 the epimysium on muscle force production" considering the possible influence of altered passive connective tissue structure and properties on the contractile apparatus in the muscle fiber and sarcomere level can provide the leverage to understand mechanisms of pathological conditions and effects of treatments for improved control over them.

Other degenerative conditions affecting muscle structure degrade gait, and early detection can help guide treatment. One example is myotonic dystrophy type 1 (the most common form of adult onset muscular dystrophy). Kim et al. have shown that functional gait assessments, such as the Functional Ambulatory Profile, correlate well with fat infiltration in lower extremity muscles and can serve as inexpensive early or longitudinal biomarkers in clinical studies.

Effects of blunt injury to the hindlimb muscles and to the lungs have been studied by Gihring et al. in lean vs. obese rats. They showed that obesity impairs the normal extracellular expansion up to 8 days past the injury, which is otherwise a normal part of the healing process. Obese rats, with fat deposits in the muscle, have a longer healing period with a prolonged period with increased pro-inflammatory cytokines and an altered satellite cell gene expression. This shows that the ECM buildup as a part of a healing process may be altered in different diseases probably not only in obesity, but also in, e.g., diabetes and maybe also in CP. Even lean children with CP have more signs of fat in the muscle compared to control when investigated with MRI (Noble et al., 2014).

\section{AUTHOR CONTRIBUTIONS}

All authors listed have made a substantial, direct, and intellectual contribution to the work and approved it for publication.

\section{ACKNOWLEDGMENTS}

The topic editors acknowledge Arda Arpak, Uluç; Pamuk and Agah Karakuzu for the Research Topic illustration.

Ateş, F., and Yucesoy, C. A. (2014). Effects of botulinum toxin type A on noninjected bi-articular muscle include a narrower length range of force exertion and increased passive force. Muscle Nerve 49, 866-878. doi: 10.1002/mus.23993

Blemker, S. S., Asakawa, D. S., Gold, G. E., and Delp, S. L. (2007). Image-based musculoskeletal modeling: applications, advances, and future opportunities. J. Magn. Reson. Imaging 25, 441-451. doi: 10.1002/jmri.20805

Bojsen-Møller, J., Schwartz, S., Kalliokoski, K. K., Finni, T., and Magnusson, S. P. (2010). Intermuscular force transmission between human plantarflexor muscles in vivo. J. Appl. Physiol. 109, 1608-1618. doi: 10.1152/japplphysiol.01381.2009

Böl, M., and Reese, S. (2008). Micromechanical modelling of skeletal muscles based on the finite element method. Comput. Methods Biomech. Biomed. Engin. 11, 489-504. doi: 10.1080/10255840701771750

Brunner, R., Jaspers, R. T., Pel, J. J. M., and Huijing, P. A. (2000). Acute and longterm effects on muscle force after intramuscular aponeurotic lengthening. Clin. Orthop. Relat. Res. 378, 264-273. doi: 10.1097/00003086-200009000-00037

Cankaya, A. O., Pamuk, U., and Yucesoy, C. A. (2021). The effects of an activation-dependent increase in titin stiffness on whole muscle 
properties using finite element modeling. J. Biomech. 116:110197. doi: 10.1016/j.jbiomech.2020.110197

Cruz-Montecinos, C., González Blanche, A., López Sánchez, D., Cerda, M., Sanzana-Cuche, R., and Cuesta-Vargas, A. (2015). In vivo relationship between pelvis motion and deep fascia displacement of the medial gastrocnemius: anatomical and functional implications. J. Anat. 227, 665-672. doi: 10.1111/joa.12370

de Bruin, M., Smeulders, M. J., Kreulen, M., Huijing, P. A., and Jaspers, R. T. (2014). Intramuscular Connective tissue differences in spastic and control muscle: a mechanical and histological study. PLOS ONE 9:e101038. doi: 10.1371/journal.pone. 0101038

de Bruin, M., Smeulders, M. J. C., and Kreulen, M. (2011). Flexor carpi ulnaris tenotomy alone does not eliminate its contribution to wrist torque. Clin. Biomechan. 26, 725-728. doi: 10.1016/j.clinbiomech.2011.03.007

de Bruin, M., Smeulders, M. J. C., and Kreulen, M. (2013). Why is joint range of motion limited in patients with cerebral palsy? J. Hand Surg. Eur. 38, 8-13. doi: $10.1177 / 1753193412444401$

Hedberg-Graff, J., Granström, F., Arner, M., and Krumlinde-Sundholm, L. (2019). Upper-limb contracture development in children with cerebral palsy: a population-based study. Dev. Med. Child Neurol. 61, 204-211. doi: $10.1111 /$ dmcn.14006

Huijing, P. A. (1999). Muscle as a collagen fiber reinforced composite: a review of force transmission in muscle and whole limb. J. Biomech. 32, 329-345. doi: 10.1016/S0021-9290(98)00186-9

Huijing, P. A. (2009). Epimuscular myofascial force transmission: a historical review and implications for new research. international society of biomechanics Muybridge award lecture, Taipei, 2007. J. Biomech. 42, 9-21. doi: 10.1016/j.jbiomech.2008.09.027

Huijing, P. A., and Jaspers, R. T. (2005). Adaptation of muscle size and myofascial force transmission: a review and some new experimental results. Scand. J. Med. Sci. Sports 15, 349-380. doi: 10.1111/j.1600-0838.2005.00457.x

Huijing, P. A., Yaman, A., Ozturk, C., and Yucesoy, C. A. (2011). Effects of knee joint angle on global and local strains within human triceps surae muscle: MRI analysis indicating in vivo myofascial force transmission between synergistic muscles. Surg. Radiol. Anat. 33, 869-879. doi: 10.1007/s00276-011-0863-1

Jaspers, R. T., Brunner, R., Baan, G. C., and Huijing, P. A. (2002). Acute effects of intramuscular aponeurotomy and tenotomy on multitendoned rat EDL: indications for local adaptation of intramuscular connective tissue. Anat. Rec. 266, 123-135. doi: 10.1002/ar.10045

Karakuzu, A., Pamuk, U., Ozturk, C., Acar, B., and Yucesoy, C. A. (2017). Magnetic resonance and diffusion tensor imaging analyses indicate heterogeneous strains along human medial gastrocnemius fascicles caused by submaximal plantarflexion activity. J. Biomech. 57, 69-78. doi: 10.1016/j.jbiomech.2017.03.028

Kaya, C. S., Bilgili, F., Akalan, N. E., Temelli, Y., Ate,ş, F., and Yucesoy, C. A. (2019). Intraoperative experiments combined with gait analyses indicate that active state rather than passive dominates the spastic gracilis muscle's joint movement limiting effect in cerebral palsy. Clin. Biomech. 68, 151-157. doi: 10.1016/j.clinbiomech.2019.06.005

Kaya, C. S., Bilgili, F., Akalan, N. E., and Yucesoy, C. A. (2020). Intraoperative testing of passive and active state mechanics of spastic semitendinosus in conditions involving intermuscular mechanical interactions and gait relevant joint positions. J. Biomech. 103:109755. doi: 10.1016/j.jbiomech.2020.109755

Kaya, C. S., Temelli, Y., Ates, F., and Yucesoy, C. A. (2018). Effects of intersynergistic mechanical interactions on the mechanical behaviour of activated spastic semitendinosus muscle of patients with cerebral palsy. J. Mech. Behav. Biomed. Mater. 77, 78-84. doi: 10.1016/j.jmbbm.2017.08.040

Le Sant, G., Nordez, A., Andrade, R., Hug, F., Freitas, S., and Gross, R. (2017). Stiffness mapping of lower leg muscles during passive dorsiflexion. J. Anat. 230, 639-650. doi: 10.1111/joa.12589

Lichtwark, G. A., Farris, D. J., Chen, X., Hodges, P. W., and Delp, S. L. (2018). Microendoscopy reveals positive correlation in multiscale length changes and variable sarcomere lengths across different regions of human muscle. J. Appl. Physiol. 125, 1812-1820. doi: 10.1152/japplphysiol.004 80.2018

Lindén, O., Hägglund, G., Rodby-Bousquet, E., and Wagner, P. (2019). The development of spasticity with age in 4,162 children with cerebral palsy: a register-based prospective cohort study. Acta Orthop. 90, 286-291. doi: $10.1080 / 17453674.2019 .1590769$
Marinho, H. V. R., Amaral, G. M., Moreira, B. S., Santos, T. R. T., Magalhães, F. A., Souza, T. R., et al. (2017). Myofascial force transmission in the lower limb: an in vivo experiment. J. Biomech. 63, 55-60. doi: 10.1016/j.jbiomech.2017.07.026

Marty, B., and Carlier, P. G. (2020). MR fingerprinting for water T1 and fat fraction quantification in fat infiltrated skeletal muscles. Magn. Reson. Med. 83, 621-634. doi: $10.1002 / \mathrm{mrm} .27960$

Noble, J. J., Charles-Edwards, G. D., Keevil, S. F., Lewis, A. P., Gough, M., and Shortland, A. P. (2014). Intramuscular fat in ambulant young adults with bilateral spastic cerebral palsy. BMC Musculoskelet. Disord. 15:236. doi: 10.1186/1471-2474-15-236

Pamuk, U., Karakuzu, A., Ozturk, C., Acar, B., and Yucesoy, C. A. (2016). Combined magnetic resonance and diffusion tensor imaging analyses provide a powerful tool for in vivo assessment of deformation along human muscle fibers. J. Mech. Behav. Biomed. Mater. 63, 207-219. doi: 10.1016/j.jmbbm.2016.06.031

Pamuk, U., and Yucesoy, C. A. (2015). MRI analyses show that kinesio taping affects much more than just the targeted superficial tissues and causes heterogeneous deformations within the whole limb. J. Biomech. 48, 4262-4270. doi: 10.1016/.j.jbiomech.2015.10.036

Pappas, G. P., Asakawa, D. S., Delp, S. L., Zajac, F. E., and Drace, J. E. (2002). Nonuniform shortening in the biceps brachii during elbow flexion. J. Appl. Physiol. 92, 2381-2389. doi: 10.1152/japplphysiol.00843.2001

Sinha, S., Sinha, U., and Edgerton, V. R. (2006). In vivo diffusion tensor imaging of the human calf muscle. J. Magn. Resonance Imaging 24, 182-190. doi: 10.1002/jmri.20593

Smeulders, M. J. C., and Kreulen, M. (2007). Myofascial force transmission and tendon transfer for patients suffering from spastic paresis: a review and some new observations. J. Electromyography Kinesiol. 17, 644-656. doi: 10.1016/j.jelekin.2007.02.002

Smeulders, M. J. C., Kreulen, M., Hage, J. J., Huijing, P. A., and van der Horst, C. M. A. M. (2004). Overstretching of sarcomeres may not cause cerebral palsy muscle contracture. J. Orthop. Res. 22, 1331-1335. doi: 10.1016/j.orthres.2004.04.006

Smeulders, M. J. C., Kreulen, M., Hage, J. J., Huijing, P. A., and van der Horst, C. M. A. M. (2005). Spastic muscle properties are affected by length changes of adjacent structures. Muscle Nerve 32, 208-215. doi: 10.1002/mus.20360

Stecco, C., Adstrum, S., Hedley, G., Schleip, R., and Yucesoy, C. A. (2018). Update on fascial nomenclature. J. Bodyw. Mov. Ther. 22:354. doi: 10.1016/j.jbmt.2017.12.015

Stecco, C., Corradin, M., Macchi, V., Morra, A., Porzionato, A., Biz, C., et al (2013). Plantar fascia anatomy and its relationship with Achilles tendon and paratenon. J. Anat. 223, 665-676. doi: 10.1111/joa.12111

Street, S. F. (1983). Lateral transmission of tension in frog myofibers: a myofibrillar network and transverse cytoskeletal connections are possible transmitters. J. Cell. Physiol. 114, 346-364. doi: 10.1002/jcp.1041140314

Tian, M., Herbert, R. D., Hoang, P., Gandevia, S. C., and Bilston, L. E. (2012). Myofascial force transmission between the human soleus and gastrocnemius muscles during passive knee motion. J. Appl. Physiol. 113, 517-523. doi: 10.1152/japplphysiol.00111.2012

Turkoglu, A. N., and Yucesoy, C. A. (2016). Simulation of effects of botulinum toxin on muscular mechanics in time course of treatment based on adverse extracellular matrix adaptations. J. Biomech. 49, 1192-1198. doi: 10.1016/j.jbiomech.2016.03.002

Westman, A. M., Dyer, S. E., Remer, J. D., Hu, X., Christ, G. J., and Blemker, S. S, (2019). A coupled framework of in situ and in silico analysis reveals the role of lateral force transmission in force production in volumetric muscle loss injuries. J. Biomech. 85, 118-125. doi: 10.1016/j.jbiomech.2019.01.025

Wilke, J., Schleip, R., Yucesoy, C. A., and Banzer, W. (2018). Not merely a protective packing organ? A review of fascia and its force transmission capacity. J. Appl. Physiol. 124, 234-244. doi: 10.1152/japplphysiol.00565.2017

Wilke, J., and Tenberg, S. (2020). Semimembranosus muscle displacement is associated with movement of the superficial fascia: an in vivo ultrasound investigation. J. Anat. 237, 1026-1031. doi: 10.1111/joa. 13283

Yilmaz, E. O., Kaya, C. S., Akdeniz-Dogan, Z. D., and Yucesoy, C. A. (2021). Longterm BTX-A effects on bi-articular muscle: Higher passive force, limited length range of active force production and unchanged intermuscular interactions. $J$. Biomech. 126:110627. doi: 10.1016/j.jbiomech.2021.110627

Yucesoy, C. A. (2010). Epimuscular myofascial force transmission implies novel principles for muscular mechanics. Exerc. 
Sport Sci. Rev. 38:128. doi: 10.1097/JES.0b013e3181e $372 \mathrm{ef}$

Yucesoy, C. A., and Huijing, P. A. (2009). Assessment by finite element modeling indicates that surgical intramuscular aponeurotomy performed closer to the tendon enhances intended acute effects in extramuscularly connected muscle. J. Biomech. Eng. 131:021012. doi: 10.1115/1.30 05156

Yucesoy, C. A., and Huijing, P. A. (2012). "8.5 - Mathematical modeling," in Fascia: The Tensional Network of the Human Body, eds. R. Schleip, T. W. Findley, L. Chaitow, and P. A. Huijing (Oxford: Churchill Livingstone), 503-509. doi: 10.1016/B978-0-7020-3425-1.0 0077-5

Yucesoy, C. A., Turkoglu, A. N., Umur, S., and Ate,ş, F. (2015). Intact muscle compartment exposed to botulinum toxin type a shows compromised intermuscular mechanical interaction: BTX-A Compromises Mechanics. Muscle Nerve 51, 106-116. doi: 10.1002/mus. 24275
Conflict of Interest: The authors declare that the research was conducted in the absence of any commercial or financial relationships that could be construed as a potential conflict of interest.

Publisher's Note: All claims expressed in this article are solely those of the authors and do not necessarily represent those of their affiliated organizations, or those of the publisher, the editors and the reviewers. Any product that may be evaluated in this article, or claim that may be made by its manufacturer, is not guaranteed or endorsed by the publisher.

Copyright (C) 2021 Yucesoy, Pontén, Valero-Cuevas, Smeulders and Simms. This is an open-access article distributed under the terms of the Creative Commons Attribution License (CC BY). The use, distribution or reproduction in other forums is permitted, provided the original author(s) and the copyright owner(s) are credited and that the original publication in this journal is cited, in accordance with accepted academic practice. No use, distribution or reproduction is permitted which does not comply with these terms. 